\title{
Profile of Children with Poor School Performance in Mumbai
}

We report on the etiology of poor school performance (PSP) in children assessed at a learning disability clinic in western India over 12 months. Specific learning disabilities (dyslexia, dysgraphia and dyscalculia) were the commonest cause of PSP $(72.76 \%)$, followed by borderline intellectual functioning $(8.94 \%)$, language barrier $(8.54 \%)$, and mental retardation $(4.88 \%)$.

Key words: Language barrier, Learning disorders, Social Class.

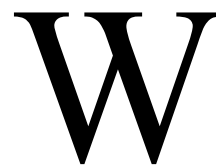

e conducted a retrospective study to analyze the profile of school children assessed for poor school performance (PSP) at our clinic from 1st April 2010 to 31st March 2011. A total of 246 children were assessed: 166 boys vs. 80 girls (male: female ratio 2.08:1). Their mean (SD) age at referral was $12.1(2.28) y$. They had scored a mean $45.23 \%$ marks ( \pm SD 11.03 , range $30-82$ ) at their last school examinations. PSP had been noticed by their school teachers and/or parents since a mean (SD) period of 3.96 (2.16)y. An overwhelming majority 234/246 (95.12\%) were studying in English-medium schools. As per Kuppuswamy's classification, $23(9.35 \%)$ belonged to upper socioeconomic class of society, 176 (71.54\%) to upper middle; and only 38 $(15.45 \%)$ to lower middle and $9(3.66 \%)$ to lower socioeconomic class of society [1].

After conducting standard clinical and psychoeducational assessments, the cause of PSP was identified in 234/246 (95.12\%) children [2,3]. The commonest diagnosis were specific learning disabilities (dyslexia/ dysgraphia/ dyscalculia) in 179/246 (72.76\%) children, followed by borderline intellectual functioning or "slow learner" (IQ score 71 to 84 ) in 22/246 (8.94\%), language barrier in $21 /$ $246(8.54 \%)$ and mental retardation in 12/246 (4.88\%) children. Attention deficit hyperactivity disorder was diagnosed, as per DSM-IV criteria, as a co-morbidity in 95/ $234(40.6 \%)$ of these diagnosed children. Also, 31/234 $(13.25 \%)$ were receiving treatment for a chronic medical condition (either, epilepsy or asthma or allergies). One child with mental retardation had co-morbid autism spectrum disorder.

All 21 children diagnosed with language barrier were studying in English-medium schools and belonged to lower middle or lower socioeconomic class of society. These 21 children, in spite of having average to above average intellectual functioning, had limited English proficiency.
Their parents had received their education (some were illiterate or semi-literate) in the vernacular medium.

In the remaining $12(4.88 \%)$ children the precise cause of PSP could not be identified and were labelled as having "learning difficulty". They were referred to a counsellor/ psychiatrist for detailed evaluation for covert emotional problems and advised to undergo remedial education and follow-up was planned after a period of one year for reassessment.

Our results suggest that even today very few children in our megacity get referred for PSP assessment and that too after a significant delay. There is an urgent need to improve awareness amongst school authorities and parents that PSP needs timely evaluation, viz. when the child is in the primary school [4]. A timely diagnosis of cause of PSP would greatly help in formulating an appropriate individual educational plan to ensure that the afflicted child can perform up to his $/$ her full potential $[3,4]$.

Acknowledgement: Dr SN Oak, Director (Medical Education and Major Hospitals, Municipal Corporation of Greater Mumbai) for granting permission to publish this manuscript.Contributors: SK conceived the paper, performed the literature review and wrote the manuscript; he will act as the guarantor of the paper. BD, AT and RS discussed the core ideas and revised the manuscript for important intellectual content. The final manuscript was approved by all authors.

Source of support: The Learning Disability Clinic at our Institute is partially funded by a research grant from Tata Interactive Systems, Mumbai. Funding: Indian Council of Medical Research, Short Term Studentship; Competing interests: None stated.

S Karande, B Doshi, A Thadhani and R Sholapurwala, Learning Disability Clinic, Department of Pediatrics, Seth GS Medical College \& KEM Hospital, Parel, Mumbai 400 012.India.karandesunil@yahoo.com

\section{REFERENCES}

1. Kumar N, Shekhar C, Kumar P, Kundu AS. Kuppuswamy's socioeconomic status scale - updating for 2007. Indian J Pediatr. 2007;74:1131-2.

2. Karande S, Kulkarni M. Specific learning disability: the invisible handicap. Indian Pediatr. 2005;42:315-9.

3. Sholapurwala RF. Curriculum based test for educational evaluation of learning disability. 1st ed. Mumbai: Jenaz Printers; 2010.

4. Karande S, Kulkarni M. Poor school performance. Indian J Pediatr. 2005;72:961-7. 